PROCEEDINGS OF THE

AMERICAN MATHEMATICAL SOCIETY

Volume 137, Number 2, February 2009, Pages 593-596

S 0002-9939(08)09665-2

Article electronically published on August 22, 2008

\title{
INCOMPARABLE PRIME IDEALS IN COMMUTATIVE RADICAL FRÉCHET ALGEBRAS
}

\author{
HUNG LE PHAM
}

(Communicated by Nigel J. Kalton)

\begin{abstract}
Let $R$ be a commutative radical Fréchet algebra having a nonnilpotent element $a$ with $a \in \overline{R a}$. Then $R$ contains a continuum of incomparable prime ideals.
\end{abstract}

In [3, J. Esterle proved the following result; it is a main ingredient in his proof that epimorphisms from $\mathcal{C}_{0}(\Omega)$ onto Banach algebras are continuous.

Theorem (Esterle). Let $R$ be a commutative radical Banach algebra. Suppose that there exists a non-nilpotent element $a \in R$ with $a \in \overline{R a}$. Then the set of prime ideals in $R$, ordered by inclusion, does not form a chain.

Thus, each algebra $R$ as in the theorem contains at least two prime ideals which are incomparable. In [1, Bouloussa extended the result to commutative radical Fréchet algebras. In this paper, we shall extend this by producing a continuum of pairwise incomparable prime ideals. This will be proved as a consequence of a result on ideals in (not necessarily commutative) radical Fréchet algebras and the existence of a continuum of "almost disjoint" subsets of $\mathbb{N}$ due to Sierpinski.

\section{Preliminaries}

More details of the following can be found, for example, in 22.

A Fréchet algebra is a topological algebra $A$ whose topology is determined by a sequence of algebra seminorms $\left(p_{n}\right)$ such that

$$
d(a, b)=\sum_{n=1}^{\infty} \frac{\min \left\{p_{n}(a-b), 1\right\}}{2^{n}} \quad(a, b \in A)
$$

is a complete metric.

Let $A$ be an algebra. Denote by $A^{\#}$ the conditional unitization of $A$ : adjoining an identity in the case where $A$ is non-unital.

Let $S$ be a subset of an algebra $A$. For $n \in \mathbb{N}$, denote by $S^{n}$ the linear span of $\left\{a_{1} \cdots a_{n}: a_{i} \in S\right\}$ (and $A^{(n)}$ the $n$-fold Cartesian product of $A$ ).

For clarity, we shall use boldface characters to denote tuples of elements; for example, we set

$$
\boldsymbol{x}=\left(x_{1}, \ldots, x_{m}\right) \quad \text { or } \quad \boldsymbol{y}=\left(y_{1}, \ldots, y_{n}\right) .
$$

Received by the editors January 23, 2008.

2000 Mathematics Subject Classification. Primary 46J05; Secondary 46J20, 46J45.

Key words and phrases. Commutative Fréchet algebra, radical, Banach algebra, prime ideal.

This research is supported by a Killam Postdoctoral Fellowship and an Honorary PIMS PDF.

(C)2008 American Mathematical Society Reverts to public domain 28 years from publication 


\section{A CONTINUUM OF INCOMPARABLE PRIME IDEALS}

Lemma 1. Let $R$ be a radical Fréchet algebra. Let $m, n \in \mathbb{N}$, and let $\left(k_{1}, \ldots, k_{n}\right) \subset$ $\mathbb{N}$. Let $I$ be a left ideal in $R$ satisfying that $I^{(m)}=\overline{R \cdot I^{(m)}}$. Suppose that $p$ is a continuous algebra seminorm on $R$ such that $I^{2^{k}-1} \not \subset \operatorname{ker} p$, where $k=k_{1}+\cdots+k_{n}$. Then, for each function $\phi: R \rightarrow \mathbb{R}^{+}$, the set of all $(\boldsymbol{x}, \boldsymbol{y}) \in I^{(m)} \times I^{(n)}$ satisfying

$$
\sum_{i=1}^{m} \phi\left(v x_{i}\right)<p\left(v y_{1}^{k_{1}} \cdots y_{n}^{k_{n}}\right)
$$

for some $v \in R^{\#}$, is dense in $I^{(m+n)}$.

Proof. Denote by $U$ the set under consideration. First, let $(\boldsymbol{a}, \boldsymbol{b})$ be arbitrary in $I^{(m+n)}$ with $\boldsymbol{a} \in R \cdot I^{(m)}$ and $p\left(b_{1}^{k_{1}} \cdots b_{n}^{k_{n}}\right) \neq 0$. Let $\boldsymbol{a}^{\prime}=\left(a_{1}^{\prime}, \ldots, a_{m}^{\prime}\right) \in I^{(m)}$ and $c \in R$ such that $c \cdot \boldsymbol{a}^{\prime}=\boldsymbol{a}$. Since $p\left(b_{1}^{k_{1}} \cdots b_{n}^{k_{n}}\right) \neq 0$, we see that (cf. [1, Lemme 1.1]), for each $r \in \mathbb{N}$, there exists $\lambda_{r} \in \mathbb{C}$ such that

$$
0<\left|\lambda_{r}\right|<\frac{1}{r} \text { and that } p\left(\left(\lambda_{r}+c\right)^{-1} b_{1}^{k_{1}} \cdots b_{n}^{k_{n}}\right)>\sum_{i=1}^{m} \phi\left(a_{i}^{\prime}\right) .
$$

Set $v_{r}=\lambda_{r}+c \in R^{\#}$ and $\boldsymbol{x}_{r}=v_{r} \cdot \boldsymbol{a}^{\prime} \in I^{(m)}$. Then

$$
\sum_{i=1}^{m} \phi\left(v_{r}^{-1} x_{r, i}\right)=\sum_{i=1}^{m} \phi\left(a_{i}^{\prime}\right)<p\left(v_{r}^{-1} b_{1}^{k_{1}} \cdots b_{n}^{k_{n}}\right),
$$

so that $\left(\boldsymbol{x}_{r}, \boldsymbol{b}\right) \in U(r \in \mathbb{N})$. We have $\lim \boldsymbol{x}_{r}=\boldsymbol{a}$, so $(\boldsymbol{a}, \boldsymbol{b}) \in \bar{U}$.

The set

$$
\left\{\boldsymbol{b} \in I^{(n)}: p\left(b_{1}^{k_{1}} \cdots b_{n}^{k_{n}}\right) \neq 0\right\}
$$

must be dense in $I^{(n)}$. For otherwise, there exist open subsets $B_{i}$ of $I(1 \leq i \leq n)$ such that $p\left(b_{1}^{k_{1}} \cdots b_{n}^{k_{n}}\right)=0$ whenever $\left(b_{1}, \ldots, b_{n}\right) \in \prod_{i=1}^{n} B_{i}$. Then we see that $p\left(b^{k}\right)=0$ whenever $b \in I$. It then follows from Nagata-Higman's theorem (see, for example, [2, Theorem 1.3.33]) that $I^{2^{k}-1} \subset \operatorname{ker} p$, contradicting the hypothesis.

Hence $U$ is dense in $I^{(m+n)}$ as claimed.

The following theorem extends [1, Theorem 1.2]; in the commutative case, it is possible to extend the proof in [1] to yield the same result.

Theorem 2. Let $R$ be a radical Fréchet algebra. Suppose that there exists a nonnilpotent element $a \in R$ such that $a \in \overline{R a}$. Then there exists a sequence $\left(a_{n}\right)$ in $R$ such that $\overline{R a_{n}}=\overline{R a}(n \in \mathbb{N})$ and such that, for each Fréchet algebra $A$ containing $R$ as a topological subalgebra,

$$
a_{j_{1}}^{k_{1}} \cdots a_{j_{n}}^{k_{n}} \notin a_{i_{1}} A+\ldots+a_{i_{m}} A
$$

for every finite sequences $\boldsymbol{i}=\left(i_{1}, \ldots, i_{m}\right), \boldsymbol{j}=\left(j_{1}, \ldots, j_{n}\right)$, and $\boldsymbol{k}=\left(k_{1}, \ldots, k_{n}\right)$ in $\mathbb{N}$ such that $\boldsymbol{i}$ and $\boldsymbol{j}$ are disjoint.

Proof. Set $I=\overline{R a}$. We see that $0 \neq a^{m} \in I^{m}$ and

$$
I^{(m)}=\overline{R \cdot I^{(m)}} \quad(m \in \mathbb{N}) .
$$

For each $k \in \mathbb{N}$, fix a continuous algebra seminorm $q_{k}$ of $R$ such that

$$
I^{2^{k}-1} \not \subset \operatorname{ker} q_{k} \text {. }
$$


Without loss of generality, we can assume that $\left(q_{k}\right)$ is an increasing sequence of seminorms defining the topology of $R$. Denote by $\Omega$ the product space $I^{\mathbb{N}}$; its topology is defined by a complete metric.

Let $d$ be any complete metric defining the topology of $R$. For each $n \in \mathbb{N}$, set $V_{n}=\{x \in I: d(a, v x)<1 / n$ for some $v \in R\}$. Then $V_{n}$ is an open subset of $I$. We see that $a \in V_{n}$, and $V_{n}$ is closed under multiplication on the left by elements in $R^{\#} \backslash R$. Hence, $V_{n}$ is dense in $\overline{R a}=I$.

For each $m, n \in \mathbb{N}$, set

$$
V_{n, m}=\left\{\left(x_{r}\right) \in \Omega: x_{r} \in V_{n}(1 \leq r \leq m)\right\} .
$$

From the previous paragraph, we see that $V_{n, m}$ is an open dense subset of $\Omega$.

For each $\boldsymbol{s}=(l, \boldsymbol{i}, \boldsymbol{j}, \boldsymbol{k})$ in $\mathbb{N} \times \mathbb{N}^{m} \times \mathbb{N}^{n} \times \mathbb{N}^{n}$, with $\boldsymbol{i}$ and $\boldsymbol{j}$ being disjoint, let $U_{\boldsymbol{s}}$ be the set of all $\left(x_{r}\right) \in \Omega$ with the property that

$$
l^{2} \sum_{t=1}^{m} q_{l}\left(v x_{i_{t}}\right)<q_{k}\left(v x_{j_{1}}^{k_{1}} \ldots x_{j_{n}}^{k_{n}}\right)
$$

for some $v \in R^{\#}$, where $k=k_{1}+\ldots+k_{n}$. By Lemma 1, this is a dense (open) subset of $\Omega$.

By the Baire category theorem, there exists $\left(a_{r}\right)$ belonging to all $U_{s}$ and $V_{n, m}$ above. Since $\left(a_{r}\right) \in V_{n, m}(n, m \in \mathbb{N})$, it follows that $a \in \overline{R a_{r}}$, and so $\overline{R a}=\overline{R a_{r}}$ $(r \in \mathbb{N})$.

Let $A$ be a Fréchet algebra containing $R$ as a topological subalgebra. Let $m, n \in$ $\mathbb{N}$, and let $\boldsymbol{i}=\left(i_{1}, \ldots, i_{m}\right), \boldsymbol{j}=\left(j_{1}, \ldots, j_{n}\right)$, and $\boldsymbol{k}=\left(k_{1}, \ldots, k_{n}\right)$ be finite sequences in $\mathbb{N}$ such that $\boldsymbol{i}$ and $\boldsymbol{j}$ are disjoint. It remains to prove that

$$
a_{j_{1}}^{k_{1}} \cdots a_{j_{n}}^{k_{n}} \notin a_{i_{1}} A+\ldots+a_{i_{m}} A .
$$

Indeed, assume toward a contradiction that $a_{j_{1}}^{k_{1}} \cdots a_{j_{n}}^{k_{n}}=a_{i_{1}} c_{1}+\ldots+a_{i_{m}} c_{m}$ for some $c_{r} \in A$. Set $k=k_{1}+\ldots+k_{n}$. The previous paragraph shows that there exists an element $v_{l} \in R^{\#}$ such that

$$
\sum_{t=1}^{m} q_{l}\left(v_{l} a_{i_{t}}\right)<\frac{1}{l} \quad \text { and } \quad q_{k}\left(v_{l} a_{j_{1}}^{k_{1}} \ldots a_{j_{n}}^{k_{n}}\right)>l \quad(l \in \mathbb{N}) .
$$

We then see that $\lim _{l \rightarrow \infty} v_{l} a_{i_{t}}=0$, so $\lim _{l \rightarrow \infty} v_{l} \sum_{t=1}^{m} a_{i_{t}} c_{t}=0$, but $\left(v_{l} a_{j_{1}}^{k_{1}} \ldots a_{j_{n}}^{k_{n}}\right.$ : $l \in \mathbb{N}$ ) can never converge in $R$ (and hence can never converge to 0 in $A$ ), a contradiction.

We now present the construction due to Sierpinski of a family $\left\{E_{\alpha}: \alpha \in \mathfrak{c}\right\}$ of infinite subsets of $\mathbb{N}$ satisfying the following properties (cf. [6]):

(i) $\mathbb{N}=\bigcup_{\alpha \in \mathfrak{c}} E_{\alpha}$, and

(ii) $E_{\alpha} \cap E_{\beta}$ is finite for each $\alpha \neq \beta \in \mathfrak{c}$.

The set $\mathbb{N}$ is isomorphic to

$$
C=\bigcup_{n=1}^{\infty}\{f:\{1, \ldots, n\} \rightarrow\{1,2\}\} .
$$

For each $f: \mathbb{N} \rightarrow\{1,2\}$, define

$$
C_{f}=\{\text { the restrictions of } f \text { to }\{1, \ldots, n\}:(n \in \mathbb{N})\} \text {. }
$$


We see that $C=\bigcup_{f: \mathbb{N} \rightarrow\{1,2\}} C_{f}$ and that $C_{f} \cap C_{g}$ is finite for each $f \neq g$. We can then map back from $C$ to $\mathbb{N}$.

Corollary 3. Let $R$ be a commutative radical Fréchet algebra. Suppose that there exists a non-nilpotent element $a \in R$ such that $a \in \overline{R a}$. Then there exists a family of prime ideals $\left(P_{\alpha}: \alpha \in \mathfrak{c}\right)$ in $R$ such that $P_{\alpha} \not \subset P_{\beta}(\alpha \neq \beta \in \mathfrak{c})$.

Proof. Let $\left(a_{n}\right)$ be a sequence in $R$ as specified in the theorem. We then see that, for each $E \subset \mathbb{N}$, there exists a prime ideal $Q_{E}$ in $R$ such that $a_{i} \in Q_{E}(i \in E)$ but $a_{j} \notin Q_{E}(j \notin E)$. Set $P_{\alpha}=Q_{E_{\alpha}}(\alpha \in \mathfrak{c})$, where $\left(E_{\alpha}: \alpha \in \mathfrak{c}\right)$ is the Sierpinski family of subsets of $\mathbb{N}$ constructed in the previous paragraph. Then $\left(P_{\alpha}\right)$ is the desired collection of prime ideals in $R$.

\section{ACKNOWLEDGMENTS}

The author would like to thank Professor Anthony To-Ming Lau for his kind support and encouragement during this research. He would also like to thank the referee for pointing out an error in the previous version of the paper.

\section{REFERENCES}

[1] S. H. Bouloussa, Caractérisation des algèbres de Fréchet qui sont des anneaux de valuation, J. London Math. Soc. (2) 25 (1982), 355-364. MR653393 (83f:46065)

[2] H. G. Dales, Banach algebras and automatic continuity, London Mathematical Society Monographs, vol. 24, The Clarendon Press, Oxford University Press, New York, 2000. MR 1816726 (2002e:46001)

[3] J. R. Esterle, Theorems of Gel'fand-Mazur type and continuity of epimorphisms from $\mathcal{C}(K)$, J. Functional Analysis 36 (1980), 273-286. MR.571409 (81m:46068)

[4] J. R. Esterle, Elements for a classification of commutative radical Banach algebras, in Radical Banach Algebras and Automatic Continuity, Lecture Notes in Mathematics, Vol. 975 (1983), 4-65, Springer, Berlin, Heidelberg, and New York. MR697578 (84h:46064)

[5] H. L. Pham, The continuity of homomorphisms and derivations from Banach algebras, Ph.D. thesis, University of Leeds, 2005.

[6] B. H. Williams, Combinatorial Set Theory, North-Holland Pub. Co., Amsterdam, New York, 1977.

Department of Mathematical and Statistical Sciences, University of Alberta, Edmonton, Alberta T6G 2G1, Canada

E-mail address: hlpham@math.ualberta.ca 